\title{
9 ESTILOS DE VIDA E BEM-ESTAR DE ESTUDANTES DO ENSINO SUPERIOR
}

\author{
| Carminda Morais ${ }^{1}$; Isabel Amorim²; Cândida Cracel Viana ${ }^{3}$; Salomé Ferreira ${ }^{4}$ |
}

\section{RESUMO}

CONTEXTO: O estudo dos estilos de vida no ensino superior tem especificidades decorrentes da interpenetração de múltiplos fatores, colocando-se a saúde e bem-estar dos jovens na agenda política das instituições.

OBJETIVO(S): Avaliar estilos de vida e bem-estar psicológico dos estudantes do IPVC; Comparar os estilos de vida e o bem-estar psicológico dos estudantes do sexo feminino e masculino; Correlacionar os estilos de vida e bem-estar psicológico.

MÉTODOS: Estudo quantitativo, correlacional e transversal. Recorreu-se a questionários de caraterização sociodemográfica, Bem-estar Psicológico (QGBEP-R) e Estilo de Vida FANTASTICO, a traduzidos e validados para a população portuguesa

RESULTADOS: Dos 291 respondentes, a maioria é do sexo feminino (80,8\%), média de idade 22,49 \pm 7,035 anos, 44,3\% encontra-se deslocado da residência, 11,7\% considera ter vícios/dependências. A maioria considera o estilo de vida "Muito Bom" (52,6\%). No item "Família/Amigos" 82,2\% responde "não tem/tem às vezes" com quem falar coisas importantes", 76,5\%" não deu/às vezes deu e recebeu afetos" e 61,5\% "não se considera membro de um grupo comunitário" Da análise das dimensões do "Fantástico" e o sexo, verifica-se que as estudantes obtêm maior pontuação nos itens "família/amigos", "tabaco" e no "comportamento sexual e de saúde". Os estudantes pontuam melhor na "atividade física/associativismo", "sono" e na "introspeção". Relativamente ao bem-estar, o valor medio foi de $24,204+4,920$, sendo superior nos rapazes. Bem-estar Psicológico e Estilos de Vida encontram-se positivamente correlacionados.

CONCLUSÕES: A maioria dos estudantes perceciona o estilo de vida "Muito bom". Estilos de vida e Bem-Estar Psicológico estão positivamente associados, com fortes interpenetrações de género, convocando a intervenção efetiva.

PALAVRAS-CHAVE: Estilo de vida saudável; Educação superior

\section{RESUMEN}

\section{"Estilos de vida y bienestar de los estudiantes universitarios"}

CONTEXTO: El estudio de los estilos de vida en la enseñanza universitaria reviste especificidades derivadas de múltiples factores, siendo la salud y el bienestar de los jóvenes puntos importantes en la agenda política de las instituciones.

OBJETIVO(S): Evaluar los estilos de vida y bienestar de los alumnos del IPVC. Comparar y correlacionar estilos de vida y bienestar psicológico de alumnos y alumnas.

METODOLOGÍA: Estudio de tipo cuantitativo, analítico y transversal. Se utilizaron un cuestionario sociodemográfico, de bienestar psicológico (QGBEP-R) y el Estilo de Vida FANTÁSTICO, traducidos y validados para la población portuguesa.

RESULTADOS: De los 291 que respondieron, la mayoría es del sexo femenino (80,8\%), promedio de edad $22,49 \pm 7,035$ años, el $44,3 \%$ se encuentra desplazado del área de residencia, el $11,7 \%$ considera tener dependencias, el tabaco el más frecuente. La mayoría considera el estilo de vida "Muy Bueno" (52,6\%). Del análisis de las dimensiones del "Fantástico" y el sexo, las estudiantes obtienen mayor puntuación en los ítems "familia / amigos", "tabaco" y en el "comportamiento sexual y de salud". Los estudiantes puntuan mejor en la "actividad física / asociativismo", "sueño" y "introspección". En relación al bienestar, el valor medio fue de 24,204+4,920, siendo superior en los chicos. Ambos, bienestar psicológico y estilos de vida están positivamente relacionados.

CONCLUSIONES: La mayoría de los estudiantes perciben el estilo de vida como "Muy bueno". Los estilos de vida y bienestar psicológico están positivamente asociados, con fuertes interpretaciones de género, solicitando una intervención eficaz.

\section{DESCRIPTORES: Estilo de vida saludable; Educación superior}

\begin{abstract}
"Higher education students' lifestyles and welfare"

BACKGROUND: The study of lifestyles in higher education has specificities due to multiple factors, being both welfare and lifestyles of young people, present on the political agenda of the institutions.

AIMS: Evaluate IPVC students' lifestyles and psychological welfare; Compare lifestyles and psychological welfare of female and male students. Correlate both lifestyles and psychological welfare.

METHODS: Quantitative, analytical and cross-sectional study. A questionnaire with a sociodemographic characterization, psychological welfare (QGBEP-R) and the FANTASTICO lifestyle were used, translated and validated for the Portuguese population.

RESULTS: Of the 291 respondents, the majority are female (80.8\%), mean age is $22.49 \pm 7.035$ years, $44.3 \%$ are from the area of residence, $11.7 \%$ consider having addictions, the most frequent tobacco. Most consider their lifestyle "Very Good" (52.6\%). In the item "Family / Friends" $82.2 \%$ answered "do not have / have sometimes" to the question with whom to speak important things" $76.5 \%$ "did not give / sometimes gave and received affections" and $61.5 \%$ "did not consider themselves members of a community group and / or self-help group". From the analysis of the dimensions of the FANTASTICO and the sex, the students scored higher on the "family / friends" items, "tobacco" and "sexual and health behaviour". The students scored better in "physical activity / associativism", "sleep" and in "introspection". Concerning welfare, the mean value was $24,204+4,920$, being higher in boys.
\end{abstract}

\section{KEYWORDS: Healthy lifestyle; Higher education}

Submetido em 30-12-2018

Aceite em 25-03-2019

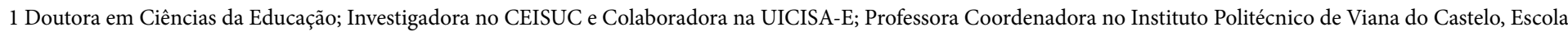
Superior de Saúde, Viana do Castelo, Portugal, carmindamorais@ess.ipvc.pt

2 Doutora em Saúde Mental; Investigadora na UICISA-E; Professora Coordenadora no Instituto Politécnico de Viana do Castelo, isabelamorim@ess.ipvc.pt

3 Mestre em Ciências da Educação-Especialização em Promoção da Saúde; Professora Adjunta no Instituto Politécnico de Viana do Castelo, candidaviana@ess.ipvc.pt

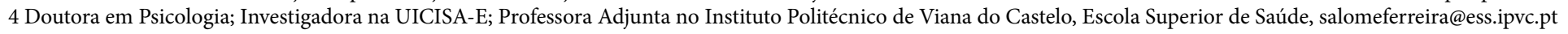

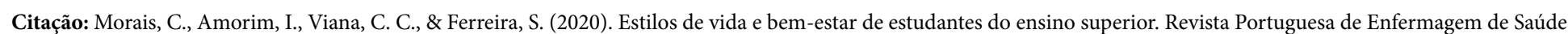
Mental (Spe7), 57-64. 


\section{INTRODUÇÃO}

Este artigo surge na sequência dos desenvolvimentos inerentes à integração do IPVC no Programa da Rede Ibero-americana das Universidades Promotoras da Saúde.

Entende-se por Instituições de Ensino Superior Promotoras da Saúde (IESPS), as organizações que desenvolvem uma cultura organizacional norteada pelos valores e princípios inerentes ao movimento global da promoção da saúde com o fim de assegurar o desenvolvimento humano e melhorar a qualidade de vida, não só das pessoas que aí trabalham e estudam como também melhorar os múltiplos contextos de vida, do trabalho, das famílias e da comunidade. Esta perspetiva assenta nas premissas da saúde positiva, pela promoção da interação de fatores psicossociais facilitadores do alcance de auto nível em bem-estar psicológico, aspeto profundamente enraizado com a saúde mental. Trata-se de reunir esforços no sentido de concretizar o preconizado em diversas orientações políticas, quer nacionais, quer internacionais, com enfoque para a carta de Otawa. Os fatores que, de uma forma ou de outra, influenciam a saúde, designados por determinantes da saúde, são inúmeros, pelo que a Organização Mundial de Saúde considera ser necessário pensar sobre a saúde, desde as suas políticas, ao ambiente e aos estilos de vida saudável e da necessidade de orientação para a promoção da saúde e prevenção da doença.

Sensibilizar as pessoas para a adoção de estilos de vida saudáveis é muito importante. A adoção de um estilo de vida saudável deve ser vista como uma oportunidade da pessoa e da comunidade, empoderando-os assim, no sentido da adotação uma atitude salutogénica nos múltiplos contextos de vida. Nesta perspetiva o bem-estar é parte integrante da conceção de saúde. Por sua vez, o conceito de bem-estar psicológico articula-se com o de saúde mental, que inclui outras dimensões positivas como a autonomia, a perceção de autoeficácia ou a competência

Nos termos propostos por Antonovski (1987), as IESPS saudáveis inscrevem-se numa perspetiva salutogénica, em que a saúde é entendida como um bem maior, suscetível de ser criada e ser vivida nos contextos quotidianos das pessoas. Ainda de acordo com Tsouros (2013), os contributos destas instituições geram potencial incrementado de saúde de diferentes formas, nomeadamente: i) pelo facto de estas abrangerem um público alargado e orientado para aspetos da vida distintos (aprender, trabalhar, socializar e usufruir de tempos livres e de lazer), utilizar serviços variados (alojamento, transportes, refeitórios, entre outros), que por este facto escamoteiam um potencial de proteção e promoção do bem-estar e da saúde de estudantes, docentes e funcionários e da comunidade envolvente que pode ser desenvolvida através de políticas e práticas; ii) Os estudantes, pela formação diversificada, poderão ser no futuro profissionais, decisores políticos, entre outros, com capacidade para influenciar as determinantes sociais da saúde; iii) de acordo com o guia das universidades saudáveis e outras IESPS, estas incorparam a promoção de saúde no seu projeto educativo e de trabalho.

Com base nestes pressupostos, as instituições envolvidas comprometem-se com referenciais de boas práticas em Educação pelos Pares e Promoção da Saúde em contexto escolar e recreativo, envolvendo, entre outros, jovens estudantes.

Neste sentido o Instituto Politécnico de Viana do Castelo (IPVC), tem potencial para se constituir como uma instituição promotora de saúde, comprometida com a promoção da literacia em saúde conducente à tomada de decisão consciente e intencional na adoção de estilos de vida e bem-estar adequados. Este processo de integração assenta no modelo PEER-IESS (Education Engagement and Evaluation Research -Instituições de Ensino Superior Salutogénicas), de Brito e Mendes (2009), que por sua vez tem por base os pressupostos teórico-metodológicos de intervenção no modelo PRECED- PROCEED (Green \& Kreuter 1991).

Este modelo envolve várias fases, designadamente a fase de diagnóstico epidemiológico, onde se inscreve o presente estudo, no âmbito da avaliação dos estilos de vida e bem-estar psicológico.

Os estilos de vida (EV) constituem uma das principais determinantes da saúde, em particular quando perspetivados em termos de prevenção. Constituem uma "variável dependente da definição de risco, ou seja, não se definem por si só, mas em relação com riscos subsequentes" (Silva, 2017, p. 73)

Cockerham (2012) desenvolveu um conceito holístico de estilos de vida, e por conseguinte, associados à saúde das comunidades e que envolve aspetos diversificados como educação, ocupação, rendimentos, englobando posteriormente o estatuto socioeconómico. Os estilos de vida são influenciados por múltiplos fatores, designadamente por valores, cultura, motivação e oportunidades.

Os estilos de vida no ensino superior revestem-se de 
especificidades decorrentes da interpenetração de uma multiplicidade de fatores biopsicossociais e culturais, numa etapa de vida, frequentemente, marcada pela separação física da família. Estes são vistos como maneiras de agir, pensar e sentir, constituindo-se como traves mestras da vida e da ação humana e simultaneamente uma alavanca na construção e promoção da saúde e bem-estar.

Por sua vez, definir bem-estar é difícil, uma vez que pode ser influenciado por variáveis tais como idade, sexo, nível socioeconómico e cultura. Para Ryff e Keyes (1995) o bem-estar será um conceito multidimensional composto por seis componentes de funcionamento psicológico positivo: avaliação positiva de si mesmo e do período anterior de vida (autoaceitação), um sentido de crescimento contínuo e desenvolvimento como uma pessoa (crescimento pessoal), acreditar que a vida possui objetivo (sentido) e significado (sentido de vida), possuir relações de qualidade com outros (relações positivas com outros), capacidade de manejar efetivamente a vida e o mundo ao redor (domínio do ambiente), e sentido de autodeterminação (autonomia). Assim o bem-estar psicológico pode ser visto como os recursos psicológicos que a pessoa detém (processos cognitivos, afetivos e emocionais) que contribuem para um bom estado de saúde física e psicológica (Ryff, 2013).

Assim, este projeto de promoção da saúde, assente nos pressupostos de empoderamento dos cidadãos para a participação na (co)construção dos seus projetos de saúde, visa, não só a adoção de estilos de vida saudáveis e bem-estar psicológico, como compromete os estudantes com o presente e futuro da sociedade.

\section{MÉTODOS}

O desenho de investigação assentou nos pressupostos enunciados, sendo que este estudo tem como objetivos: avaliar os estilos de vida e o bem-estar psicológico e dos estudantes do IPVC; comparar os estilos de vida e o bem-estar entre os estudantes do seco feminino e masculino; correlacionar os estilos de vida e bem-estar psicológico.

Realizou-se um estudo quantitativo, transversal e analítico-correlacional, utilizando-se na recolha de dados três instrumentos: um questionário de caraterização sociodemográfico; o Questionário Estilo de Vida FANTASTICO versão traduzida e adaptada de Lange e Vio (2006), Life-style Assessment Questionnaire (LAQ) (Hettler, 1982) - "O meu estilo de vida" validada para a população portuguesa (Silva, Brito \& Amado,2014); e o Psychological General Well-Being Index-Short version (PGWB-S) Grossi et al. (2006) versão traduzida e adaptada para a população portuguesa por Rainho et al. (2018).

De acordo com Silva et al., (2014) o Questionário FANTASTICO é um instrumento de autopreenchimento composto por 30 itens, de resposta fechada, e explora 10 domínios da componente física, psicológica e social dos estilos de vida. Cada domínio identifica-se com a sigla "FANTASTICO": F - Família e Amigos (itens 1,2); A - Atividade Física/Associativismo (itens 3,4,5); $\mathrm{N}$ - Nutrição (itens 6,7,8); T - tabaco (itens 9,10); A - Álcool e outras drogas (itens11,12,13,14,15,16); S - Sono/stress (itens 17,18,19); T - Trabalho/Tipo de personalidade (itens 20,21,22); I - Introspeção (itens 23,24,25); C - Comportamentos de saúde sexual (itens 26,27,28); O - Outros comportamentos (itens 29,30). Os itens possuem três opções de resposta, 0,1 ou 2 pontos e a soma de todos os pontos, resultante de todos os domínios varia ente 0 e 120 pontos e quanto menor a pontuação obtida, maior será a necessidade de reequacionar os estilos de vida. A interpretação dos resultados é efetuada com base em 4 categorias, a saber: "Excelente" (103-120), indica que o estilo de vida exerce uma elevada influência sobre a saúde; "Muito Bom" (85-102), indica que o estilo de vida exerce uma adequada influência para a saúde; "Bom" (73-84), indica que o estilo de vida traz muitos benefícios para a saúde; "Regular" (57-72), indica que o estilo de vida proporciona algum benefício para a saúde mas com alguns riscos; "Necessita melhorar" (0-46), indica que o estilo de vida apresenta muitos fatores de risco para a saúde. Para a avaliação do bem-estar utilizou-se a versão abreviada do questionário Bem-estar Psicológico (QGBEP-R) constituída por 6 itens. Este questionário assume um formato tipo Likert, com seis alternativas de resposta: a cotação dos itens varia entre 0 e 5 pontos, com a cotação invertida nos itens 2,3 e 6 . O nível de bem-estar psicológico pode assim variar entre o mínimo de 0 e o máximo de 30 , correspondendo o valor 0 ao nível mínimo e 30 ao nível máximo de bemestar psicológico. Relativamente aos procedimentos, numa primeira fase todos os estudantes, em sede de sala de aula, foram informados dos objetivos, natureza e âmbito da participação, garantidas a confidencialidade dos dados recolhidos, privacidade e anonimato, sendo também assegurada a possibilidade de os mesmos poderem desistir do estudo a qualquer momento. 
Após aceitarem participar no estudo, através das suas contas institucionais de correio eletrónico, foi disponibilizado um link com acesso aos questionários para preencher. A recolha decorreu entre fevereiro e abril de 2018.

Participaram no estudo 291 estudantes do $1^{\circ}$ ano das Licenciaturas em Enfermagem e de Educação Básica, das Escolas Superiores de Saúde (ESS) e de Educação (ESE) do IPVC, Curso Superior Profissional de Termalismo e Bem-estar e Mestrados em Enfermagem.

Para o tratamento de dados foi utilizado o programa SPSS, versão 21, recorrendo-se à estatística descritiva e inferencial, respeitando os pressupostos de normalidade e da mensuração das variáveis, sendo o nível de significância definido de 5\%.

Tendo a presente investigação resultado da extensão do estudo Estilos de Vida em Estudantes no Ensino Superior na Zona Centro (Silva, 2017) a várias instituições de Ensino Superior, designadamente ao IPVC, assumiram-se os pressupostos aprovados pela Comissão de Ética da The Health Sciences Research Unit: Nursing (UICISA-E). Foram, assim, respeitadas as recomendações das Declarações de Helsínquia e das revisões de Tóquio, Veneza, Hong Kong, Sommerset West, Edimburgo e Seul para investigações envolvendo pessoas.

\section{RESULTADOS}

Integraram o estudo 291 estudantes do Ensino Superior (estudantes do $1^{\circ}$ ano da ESS e ESE), havendo 84,2\% a frequentarem uma licenciatura, $11,4 \%$ a frequentar um mestrado e $4,5 \%$ um CTeSP. A grande maioria dos estudantes $(80,8 \%)$ são do sexo feminino e 56 estudantes (19,2\%) são do sexo masculino, com idades compreendidas entre os 18 e os 56 anos, cuja média de idades é de 22,73 anos e um desvio padrão de 6,683.

A maioria destes estudantes não trabalha $(67,0 \%)$ e uma percentagem de $33,0 \%$ refere trabalhar, sendo que $16,8 \%$ trabalha a tempo integral e $15,8 \%$ a tempo parcial. A maior parte dos estudantes mantêm-se na sua residência habitual $(55,7 \%)$, enquanto 129 estudantes $(44,3 \%)$ estão deslocados da sua residência habitual.

A grande maioria $(88,3 \%)$ refere não ter vícios ou dependências e 34 estudantes $(11,7 \%)$ considera ter vícios/dependências, sendo os mais representativos o consumo de tabaco ( 21 estudantes - 7,2\%), o consumo de café (4 estudantes - 1,4\%) e o consumo de açúcares e doces (3 estudantes - 1,0\%).
Os outros vícios/dependências (bebidas energéticas e fast food, dinheiro, cigarros eletrónicos, sair à noite, telemóvel, comida) são todos referidos com a mesma percentagem $(0,3 \%)$.

Relativamente aos resultados obtidos através do FANTASTICO, os resultados maioritariamente apontam para muito bom $(52,2 \%, n=153)$, bom $(26,8 \% n=78)$, excelente $(13,7 \% \mathrm{n}=40)$. Dito de outro modo, $93,1 \%$ $(n=271)$ perceciona o seu estilo de vida como adequado e $6,9 \%(n=20)$ como não adequado.

Verifica-se que as áreas de estilo de vida mais comprometidas, em que o valor médio obtido se distancia mais do valor máximo são nos domínios "trabalho/ tipo de personalidade", "atividade física e associativismo", "nutrição" e "sono e stresse", sempre com piores resultados para as respondentes do sexo feminino. No entanto, identifica-se nos respondentes masculinos também uma área comprometida que é "comportamento de saúde e sexual".

$\mathrm{Na}$ análise da influência do sexo nos estilos de vida dos estudantes, é possível verificar diferenças estatisticamente significativas no que diz respeito aos itens "família/amigos" com as estudantes a obterem maior pontuação ( $\mathrm{t}=-3,051 ; \mathrm{p}=0,003)$, "tabaco" $(\mathrm{t}=-2,587$; $\mathrm{p}<0,012)$ no "álcool e drogas" $(\mathrm{t}=-3,615 ; \mathrm{p}<0,001)$, e "comportamento sexual" ( $\mathrm{t}=-2,795 ; \mathrm{p}=0,006)$.

Os estudantes do sexo masculino pontuam melhor na "atividade física/associativismo" ( $\mathrm{t}=2,629 ; \mathrm{p}=0,009)$, sono $(t=3,511 ; p=0,001)$ e na "introspeção" $(t=-2,358$; $\mathrm{p}=0,019)$, como consta da Tabela 1 .

Relativamente ao bem-estar psicológico, verifica-se um o valor medio de $18,13 \pm 4,920$, configurando-se com um valor satisfatório. Quando se faz a comparação entre sexos é possível verificar que os rapazes apresentam um valor superior $(19,79+5,450)$ relativamente às raparigas $(17,74+4,712)$ com diferenças estatisticamente significativa $(\mathrm{t}=2,835, \mathrm{p}=0,005)$ como consta da Tabela 2.

Quando se procede ao estudo da correlação entre as variáveis em estudo (Bem-estar psicológico e Estilos de vida) verifica-se uma correlação positiva alta com elevado significado estatístico $(R p=0,706 ; p<0,001)$, ou seja, a um estilo de vida melhor corresponde melhor perceção de bem-estar psicológico. 
Tabela 1 - Resultados das dimensões do FANTASTICO segundo o sexo, valores do teste t de Student e significância estatística

\begin{tabular}{|c|c|c|c|c|c|}
\hline & Sexo feminino & Sexo masculino & Total & t Student & Significância \\
\hline & $\mathrm{n}=235$ & $\mathrm{n}=56$ & $\mathrm{n}=291$ & & \\
\hline & $\mathrm{M}(\mathrm{DP})$ & $\mathrm{M}(\mathrm{DP})$ & $\mathrm{M}(\mathrm{DP})$ & $\mathrm{t}$ & $\mathrm{p}$ \\
\hline Total - "FANTASTICO" & $89,62(11,130)$ & $88,93(13,846)$ & $89,48(11,678)$ & $-0,346$ & 0,730 \\
\hline Min-Máx & $58-118$ & $48-112$ & $48-118$ & & \\
\hline Sub-Total - “F” & $3,52(0,823)$ & $3,05(1,069)$ & $3,43(0,893)$ & $-3,051$ & 0,003 \\
\hline Min-Máx & $0-4$ & $0-4$ & $0-4$ & & \\
\hline Sub-total - "A" & $3,37(1,457)$ & $3,95(1,600)$ & $3,48(1,500)$ & 2,629 & 0,009 \\
\hline Min-Máx & $0-6$ & $0-6$ & $0-6$ & & \\
\hline Sub-Total - "N" & $3,60(1,189)$ & $3,61(1,384)$ & $3,60(1,226)$ & 0,062 & 0,950 \\
\hline Min-Máx & $0-6$ & $0-6$ & $0-6$ & & \\
\hline Sub-Total - “T” & $3,58(0,9049)$ & $3,07(1,412)$ & $3,48(1,038)$ & $-2,587$ & 0,012 \\
\hline Min-Máx & $0-4$ & $0-4$ & $0-4$ & & \\
\hline Sub-Total - "AA" & $11,36(0,878)$ & $10,55(1,617)$ & $11,21(1,104)$ & $-3,615$ & 0,001 \\
\hline Min-Máx & 7-12 & 5-12 & $5-12$ & & \\
\hline Sub-Total - "S" & $3,80(1,517)$ & $4,57(1,346)$ & $3,95(1,514)$ & 3,511 & 0,001 \\
\hline Min-Máx & $0-6$ & $0-6$ & $0-6$ & & \\
\hline Sub-Total - “TT" & $3,39(1,346)$ & $3,63(1,229)$ & $3,44(1,326)$ & 1,185 & 0,237 \\
\hline Min-Máx & $0-6$ & $1-6$ & $0-6$ & & \\
\hline Sub-Total - "I" & $4,06(1,439)$ & $4,55(1,347)$ & $4,15(1,433)$ & 2,357 & 0,019 \\
\hline Min-Máx & $0-6$ & $0-6$ & $0-6$ & & \\
\hline Sub-Total - “C” & $4,33(1,285)$ & $3,80(1,212)$ & $4,23(1,286)$ & $-2,795$ & 0,006 \\
\hline Min-Máx & $0-6$ & $1-6$ & $0-6$ & & \\
\hline Sub-Total - “O” & $3,81(0,571)$ & $3,68(0,636)$ & $3,78(0,585)$ & $-1,401$ & 0,165 \\
\hline Min-Máx & $0-4$ & $2-4$ & $0-4$ & & \\
\hline
\end{tabular}

Tabela 2 - Resultados da perceção de bem-estar psicológico segundo o sexo, valores do teste t de Student e significância estatística

\begin{tabular}{|l|l|l|l|l|l|}
\hline & Sexo feminino & Sexo masculino & Total & t Student & Significância \\
\cline { 2 - 6 } & $\mathrm{n}=235$ & $\mathrm{n}=56$ & $\mathrm{n}=291$ & & \\
\cline { 2 - 6 } & $\mathrm{M}(\mathrm{DP})$ & $\mathrm{M}(\mathrm{DP})$ & $\mathrm{M}(\mathrm{DP})$ & $\mathrm{t}$ & $\mathrm{p}$ \\
\hline Bem-estar Psicológico & $17,74(4,712)$ & $19,79(5,450)$ & $18,13(4,920)$ & 2,835 & 0,005 \\
\hline Min-Máx & $4-29$ & $1-30$ & $1-30$ & & \\
\hline
\end{tabular}

\section{DISCUSSÃO}

Os resultados obtidos referem-se maioritariamente a estudantes da licenciatura e predominantemente do sexo feminino. Estes factos poderão ser explicados, pelo menos em grande medida, por um lado, por o ensino superior, designadamente nas áreas da saúde e da educação, continuar a ser maioritariamente frequentado por mulheres e, por outro, os cursos de licenciatura constituem o "core" desta instituição e funcionam maioritariamente em regime presencial, o que poderá favorecer a participação no estudo. Acresce ainda o facto de o estudo ser liderado por uma equipa de investigação de uma Escola Superior de Saúde e naturalmente, nesta fase inicial, pode constituir-se um fator igualmente favorável à adesão maioritária da população feminina. Esta é uma realidade a merecer atenção acrescida, dado que se pretende integrar progressivamente outras Unidades Orgânicas da Instituição, onde as questões do género estão muito presentes. Tanto mais que o estudo dos estilos de vida, constitui apenas uma parte do diagnóstico epidemiológico, segundo o modelo PEER-IESS (Brito \& Mendes, 2018), a suportar a intervenção que se perspetiva numa lógica de pesquisa - ação participada em saúde (ibidem; Morais, Brito e Tomás, 2018).

Um aspeto a merecer atenção acrescida, designadamente em termos de intervenção, prende-se com o facto de 44,3\% da população se encontrar deslocado da sua residência habitual. 
Reconhecidamente, trata-se de um território em que as acessibilidades estão comprometidas com potencial impacto, particularmente vivido numa fase que, frequentemente, coincide com a entrada no ensino superior. Dito de outro modo, trata-se de uma etapa de adaptação frequentemente fomentadora de crises ou problemas emocionais, implicando mudanças de hábitos e estilos de vida por parte dos estudantes (Gonçalves e Carvalho, 2007; Tassini, Vale, Candico \& Bachur, 2017). Urge que, com os estudantes, por antecipação, sejam criadas condições promotoras de respostas adaptativas às transições.

Dos resultados obtidos, salienta-se a perceção de um estilo de vida "Bom e/ou Muito Bom", apontando para uma previsibilidade de maior adequação dos estilos de vida sobre a saúde. No entanto, salienta-se o facto de $6,9 \%$ dos estudantes os percecionar (estilos de vida) como inadequados. Apesar de esta frequência ser bastante inferior ao encontrado em outros estudos nacionais (Monteiro, Rainho e Barroso, 2018; Silva, 2016) e internacionais (Bonfim, Mafra, Gharib, De-Carli \& Zafalon, 2017), implica a necessidade de uma intervenção específica a esta fase da vida.

Relativamente às dependências referenciadas, surge o tabaco como mais frequente, o que é corroborado por outros estudos (Sequeira, Carvalho, Borges e Sousa, 2013). Importa futuramente perceber se este hábito se agrava ou não com a entrada no ensino superior.

Em termos de relações entre os estilos de vida avaliados pelo FANTASTICO e o sexo, constata-se que não há diferenças estatisticamente significativas em relação ao score global. Contudo, os resultados obtidos na maioria dos domínios apontam para uma indelével influencia das questões de género. Neste contexto, importa referir que as estudantes evidenciam melhores resultados, com diferenças estatisticamente significativas, nos aspetos relacionados com "família/amigos", "tabaco", "álcool e drogas" e "comportamentos sexual", parecendo evidenciar que as raparigas poderão vivenciar e expressar os afetos, pelo menos de forma mais explícita, e estão menos expostas a riscos para a saúde relacionados com o consumo destas substâncias e comportamentos sexuais inadequados. Estes resultados são corroborados pelo estudo de Ferreira (2018) mas contrariam os obtidos por Rodriguez-Gázquez, ChaparroHernandez e González-López (2016). Relativamente aos domínios "atividade física/associativismo", "sono e stresse" e "introspeção" existem igualmente diferenças estatisticamente significativas, sendo que os rapazes apresentam melhores resultados, traduzidos pelas médias mais elevadas, aspetos que são corroborados pelo estudo de Ferreira (2018). Estes resultados parecem apontar para o maior envolvimento dos rapazes em atividades da esfera pública (concretamente atividades físicas e associativismo) e maior perceção da capacidade para lidar com as emoções, sentimentos, gestão do stress e melhor qualidade de sono. Estes resultados são fortemente mediados pelas questões culturais e relações sociais de género, mas que poderão ter eventual tradução na forma de viver os tempos livres e de lazer. No que se refere ao bem-estar psicológico, a média obtida é ligeiramente mais baixa em comparação com outros estudos em instituições de ensino superior (Silva, 2016; Monteiro, Rainho e Barroso, 2018). Contudo, a par com as diferenças das dimensões dos estilos de vida, verifica-se uma associação positiva alta entre os estilos de vida e o bem-estar psicológico, sendo as estudantes do sexo feminino as que obtêm valores médios substancialmente mais baixos do que os estudantes do sexo masculino. Nesta medida, a comunidade educativa confronta-se com vários desafios, designadamente o combate das desigualdades de género, em prol da maximização do funcionamento psicológico positivo, na globalidade das componentes defendidas por Ryff e Keyes (1995).

Neste contexto, evidencia-se a necessidade de estudos longitudinais de modo a compreender a evolução dos estilos de vida numa etapa da vida marcada pela conquista de autonomia e responsabilidade, mas também de transição com ambiente de proximidade e afeto familiar, implicando a necessidade de intervenção em (co)construção e com envolvimento ativo de pares (Tassini, Vale, Candico \& Bachur, 2017).

\section{CONCLUSÕES}

Apesar da maioria dos estudantes percecionar os estilos de vida como "Muito bom/bom" e o bem-estar psicológico obtido ser satisfatório existe ainda uma proporção com algum significado a sugerir intervenções conducentes a uma promoção da saúde e do funcionamento psicológico positivo mais efetivo.

A partir das diferenças de estilos de vida e do bemestar psicológico entre rapazes e raparigas, conclui-se ainda da existência de um forte atravessamento das relações sociais de género também na vida académica. Estes aspetos, associados a um conjunto de fatores afetivos, emocionais, socioculturais e económicos, configuram determinantes sociais da saúde suscetíveis de 
ser modificados de forma intencional e crítica pelos próprios estudantes. De facto, a habilidade social combinada com o otimismo facilita não só a integração na vida académica como também atitude e determinação face à consecução dos objetivos nas várias dimensões da vida. Neste sentido este estudo constitui-se uma das primeiras etapas de um processo de mudança e transformação no âmbito da cidadania em saúde, ou seja, numa lógica em que a comunidade académica (onde os estudantes são o foco central) é atora e autora de todo o percurso.

\section{IMPLICAÇÕES PARA A PRÁTICA CLINICA}

Este estudo constitui a fase inicial do diagnóstico epidemiológico em torno dos estilos de vida dos estudantes de duas Unidades Orgânicas do Instituto Politécnico de Viana do Castelo, prevendo-se o alargamento faseado às restantes Unidades. Passar-se-á, de seguida, à fase da socialização dos resultados e construção do plano de intervenção com a comunidade educativa e órgãos de gestão. Está em jogo a inclusão e mobilização da promoção da saúde e bem-estar psicológico no projeto educativo e laboral, a equidade, a sustentabilidade e a promoção de ambientes de vida e aprendizagem saudáveis.

Desta forma, constitui-se condição para prosseguir a implementação de políticas de bem-estar e qualidade de vida na instituição. A comunidade académica, em particular os estudantes, passam a ter condições para assumir um papel ativo em todo o processo, na gestão organizacional da própria instituição e na criação de políticas de promoção da saúde e bem-estar através da sua ação, enquanto educadores pelos pares e peer- research. Esta forma de intervir é por si só promotora do desenvolvimento das competências dos enfermeiros de cuidados gerais, sobretudo no que se refere às questões da cidadania em saúde.

Perspetiva-se assim a base de reorientação de "pensar" e "fazer" a formação em enfermagem num compromisso efetivo, entre outros, com os Objetivos para o Desenvolvimento Sustentável 2020-2030, da OMS. Só assim se poderá contribuir para um "desenvolvimento científico e tecnológico igualitário, inclusivo e orientado para o futuro" (ponto 1, alínea c, da Estratégia Nacional para a Igualdade e a Não Discriminação, 2018-2030)

\section{REFERÊNCIAS BIBLIOGRÁFICAS}

Antonovsky, A. (1987). Unraveling the Mystery of Health: How People Manage Stress and Stay Well. San Francisco: Jossey Bass.

Bonfim, R.A, Mafra, M. A., Gharib, I.M., De-Carli, A. D., \& Zafalon, E. J. (2017). Factores associados ao estilo de vida fantástico de universitários brasileiros: uma análise multinível. Revista CEFAC, 19 (5), 601610. Doi: 10.1590/19820216201719518716

Brito, I., \& Mendes, F. (2018). Como estruturar um projeto de Pesquisa-Ação-Participativa em Saúde. In I. Brito (coord.), Pesquisa-Ação Participativa na (co) criação de instituições de Ensino Superior Promotoras de Saúde (77-93). Coimbra: Palimage.

Brito, I., \& Mendes, F. (2009). PEER-IESS: Instituições de Ensino Superior Salutogénicas. Coimbra: Unidade de Investigação em Ciências da Saúde.

Cockerham, W. (2012). Medical sociology. (12th ed.). Boston: Prentice Hall.

Ferreira, F.P., (2018). Contributos da pesquisa-ação participativa na transformação de uma Escola Superior de Enfermagem num contexto promotor de Saúde. (Tese de Doutoramento, Instituto de Ciências Biomédicas Abel Salazar, Universidade do Porto).

Green, L.W., \& Kreuter, M.W. (1991). Health Promotion Planning: an educational and Environmental Approach. Patient Education and Counseling, 19 (3), 298. Doi: 10.1016/0738-39991(92)90152-9

Hettler, B. (1982). Wellness promotion and risk reduction on university Campus. In M. M. Faber, \& A. M. Reinhardt (Eds.), Promoting health through risk reduction (07-238). New York: Collier Macmillan Publishers.

Monteiro, M.J.F.S.P., Rainho, M.C.A. \& Barroso, I.M.A.R.C. (2018). Bem-estar psicológico e estilo de vida saudável em estudantes da Universidade de Trásos-Montes e Alto Douro (UTAD). In I. Brito (coord.), Pesquisa-Ação Participativa na (co)criação de instituições de Ensino Superior Promotoras de Saúde (119125). Coimbra: Palimage. 
Morais, C., Brito, I., \& Tomás, C. (2018). Pesquisaação participativa na (co)construção de percursos de literacia em Saude. In I. Brito (coord.), Pesquisa-Ação Participativa na (co)criação de instituições de Ensino Superior Promotoras de Saúde (61 -76). Coimbra: Palimage.

Rodriguez-Gázquez, M., Chaparro-Hernandez, S. \& González-López, J.R. (2016). Lifestyles of nursing students from a Colombian Public University. Investigación y Educación en Enfermería, 34(1), 94-103. Doi: 10.17533/udea.iee.v34n1a11

Ryff, C. D. \& Keyes, C. L. M. (1995). The structure of psychological well-being revisited. Journal of Personality and Social Psychology, 69 (4), 719-727. Disponível em: http://midus.wisc.edu/findings/pdfs/830.pdf

Ryff, C. D. (2013). Eudaimonic well-being and health: Mapping consequences of self-realization. In A.S. Waterman (Ed.), The best within us: Positive psychology perspectives on eudamain (77-98). Washington: American Psychology Association.

Sequeira, C.; Carvalho, J. C; Borges, E. \& Sousa, C. (2013). Vulnerabilidade mental em estudantes de enfermagem no ensino superior: estudo exploratório. Journal of Nursing and Health, 3(2), 170 - 181. Disponível em: https://comum.rcaap.pt/handle/10400.26/17856
Silva, A., Brito, I. \& Amado, J. (2014). Tradução, adaptação e validação do questionário Fantastic Lifestyle Assessment em estudantes do ensino superior. Ciência \& Saúde Coletiva, 19(6), 1901-1909. Disponível em: http://www.scielo.br/scielo.php?pid=S1413$81232014000601901 \&$ script $=$ sci_abstract\&tlng=pt

Silva, A. (2017). Estilos de Vida em Estudantes do Ensino Superior.(Tese de Doutoramento, Universidade Católica Portuguesa). Disponível em: https://repositorio.ucp.pt/handle/10400.14/24196

Tassini, C., Val, G., Candido, S. \& Bachur, C. (2017). Assessment of the lifestyle of university students in the healthcare area using the Fantastic Questionnaire. International Journal of Cardiovascular Sciences, 30(2), 117-122. Doi:10.5935/23594802.20170024

Tsouros, A. (2013). City Leadership for Health and Well-being: Back to the Future. Journal of Urban Health : Bulletin of the New York Academy of Medicine, 90(supll.1), 4-13. Doi:10.1007/s11524-013-98258

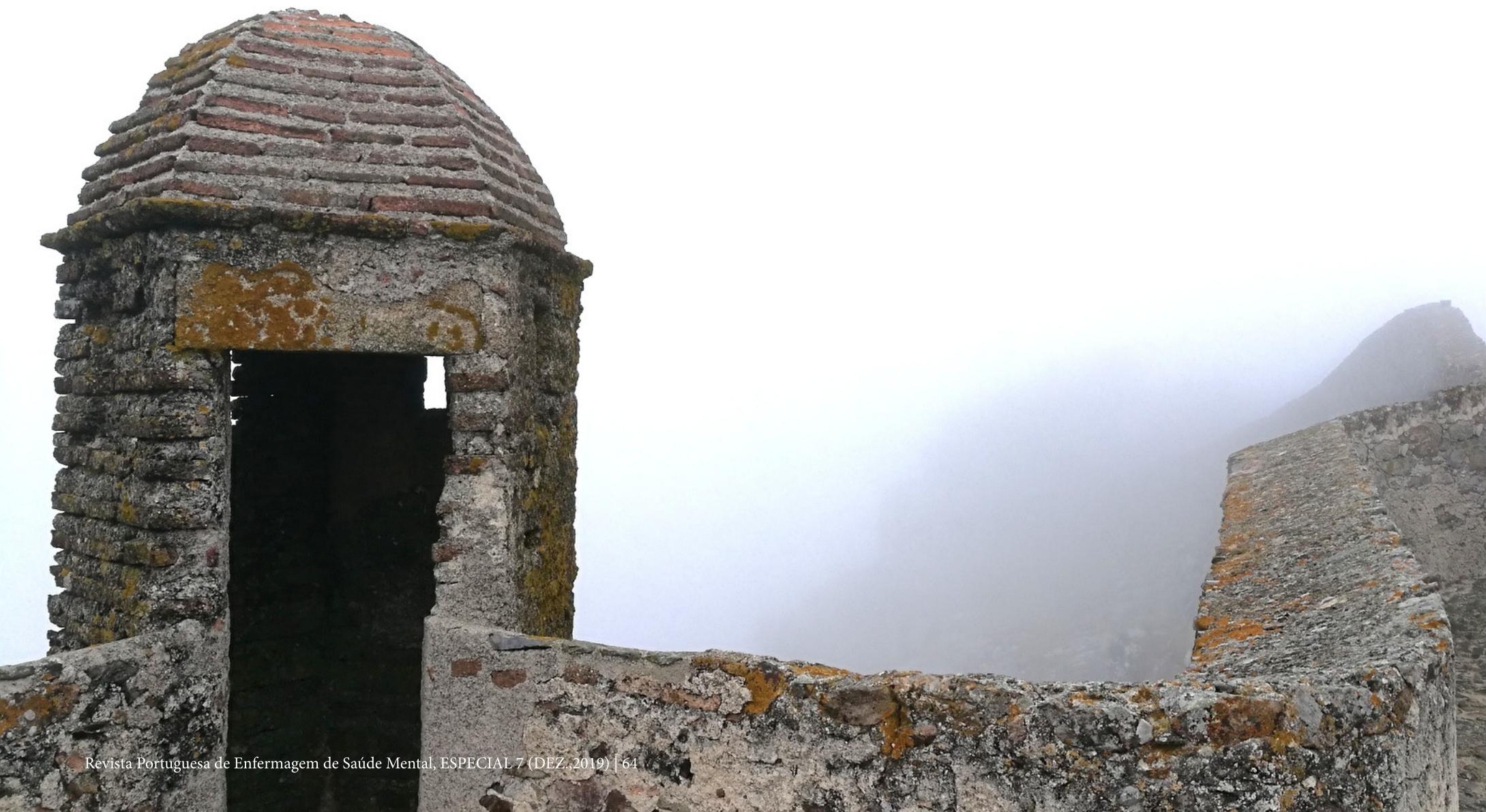

